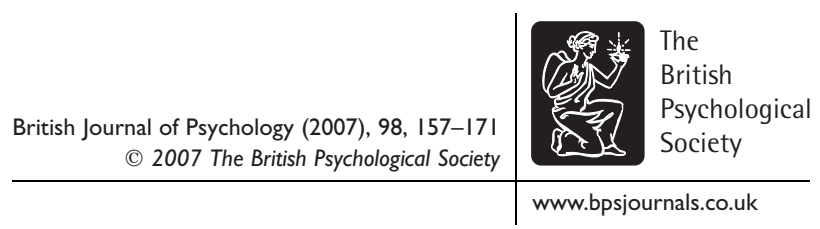

\title{
How important are linguistic factors in word skipping during reading?
}

\author{
Denis Drieghe ${ }^{\prime *}$, Timothy Desmet' and Marc Brysbaert ${ }^{2}$ \\ 'Ghent University, Belgium \\ ${ }^{2}$ Royal Holloway, University of London, UK
}

\begin{abstract}
The probability of skipping a word is influenced by its processing ease. For instance, a word that is predictable from the preceding context is skipped more often than an unpredictable word. A meta-analysis of studies examining this predictability effect reported effect sizes ranging from 0 to $13 \%$, with an average of $8 \%$. One study does not fit within this picture and reported $23 \%$ more skipping of Dutch pronouns in sentences in which the pronoun had no disambiguating value (e.g. 'Mary was envious of Helen because she never looked so good') than in sentences where it did have a disambiguating value (e.g. 'Mary was envious of Albert because she never looked so good'). We re-examined this ambiguity in Dutch using a task that more closely resembles normal reading and observed only a $9 \%$ difference in skipping of the pronoun, bringing this linguistic effect in line with the other findings.
\end{abstract}

In order to understand what determines eye guidance in text reading, many studies have focused on visual and linguistic factors to explain eye movement variables such as fixation times and saccade lengths. Whereas these investigations have led to a huge increase in our understanding of eye movements in reading (Liversedge \& Findlay, 2000; Rayner, 1978, 1998), a number of issues remain disputed (Starr \& Rayner, 2001). One of these controversies deals with the question of what precisely influences the eye guidance system when it decides not to make a saccade to the next word but to fixate the following word (i.e. word skipping). In this article, we focus on the relative contributions of visual vs. linguistic factors in this decision, an issue we will show to be at the core of the ongoing debate concerning the skipping of words in reading.

Although eye movement patterns during reading seem almost incomprehensibly complex, much can be gained from considering every eye movement as an individual decision of where and when to move the eyes. Interestingly, there is a big difference between the variables that influence these two decisions. The decision of when to move the eyes away from a word (reflected in fixation times on that word) is primarily

The first two authors contributed equally to this work.

* Correspondence should be addressed to Denis Drieghe, Department of Experimental Psychology, Ghent University, Henri Dunantlaan 2, B-9000 Ghent, Belgium (e-mail: denis.drieghe@UGent.be). 
determined by the processing ease of the word. A very robust finding is that readers will spend more time looking at a low-frequency word than at a high-frequency word (e.g. Inhoff \& Rayner, 1986; Rayner \& Duffy, 1986; Schilling, Rayner, \& Chumbley, 1998). Likewise, a word that is predictable from the preceding context will be looked at for a shorter time than a neutral word (e.g. Balota, Pollatsek, \& Rayner, 1985; Binder, Pollatsek, \& Rayner, 1999; Ehrlich \& Rayner, 1981; Rayner \& Well, 1996; Vitu, 1991). Even though visual factors can influence the gaze duration on a word (e.g. a longer word will receive a longer gaze duration; Rayner, Sereno, \& Raney, 1996), the linguistic properties of the word account for quite a large part of the variance in fixation times. On the other hand, the decision of where to move the eyes seems to be mostly determined by visual factors, such as the length of the currently fixated word and the lengths of the next words. For instance, Rayner (1979) showed that readers have a tendency to make the first fixation on a word slightly left of the centre of that word (but see White \& Liversedge, 2004 for an example of a linguistic influence on landing site).

A first indication of the disputed nature of the word skipping phenomenon is that it is difficult to place within this commonly used when/where dichotomy. Robust influences of both a low-level visual nature and a high-level linguistic nature have been shown to affect skipping behaviour. A typical low-level visual effect on word skipping is the effect of launch site: the closer the eyes are to the parafoveal word, the higher the probability that this word will be skipped on the next saccade (Kerr, 1992; Rayner et al., 1996; Vitu, O'Regan, Inhoff, \& Topolski, 1995). The most robust empirical finding in word skipping related to the characteristics of the word itself is the effect of word length: readers tend to skip short words more often than long words (e.g. Rayner, 1979; Rayner \& McConkie, 1976; Vitu et al., 1995). Interestingly, this observation has also been made when readers are asked to fake eye movements while scanning through z-strings. Based on the similarities between the skipping patterns of string scanning and normal reading Vitu et $a l$. concluded that predetermined oculomotor strategies are an important determinant of eye movement control in normal reading. However, these conclusions have been questioned by subsequent research (Rayner \& Fischer, 1996), and experimental manipulations have been shown to affect skipping of z-strings and normal words in different ways (Drieghe, Brysbaert, \& Desmet, 2005). In this latter study, Drieghe et al. observed that whereas adding an extra blank space after a z-string increased the fixation probability of that string by $10 \%$, the same manipulation had no effect whatsoever on the fixation probability of an actual word. Observations such as these cast serious doubts on the generalizability of the findings on skipping in z-string scanning to normal reading.

The fact that short words are skipped more often than long words could be due to either the length of the word or the processing ease of the word (many short words are high-frequency words or syntactic function words). Processing ease has also been shown to influence skipping behaviour when word length is controlled for. A word that is predictable from the preceding context is skipped more often than a word that is not predictable (e.g. Balota et al., 1985; Drieghe, Brysbaert, Desmet, \& Debaecke, 2004; Ehrlich \& Rayner, 1981; Rayner, Binder, Ashby, \& Pollatsek, 2001; Rayner \& Well, 1996) and a high-frequency word is skipped more often than a low-frequency word (e.g. Henderson \& Ferreira, 1993; Radach \& Kempe, 1993; Rayner \& Fischer, 1996; Rayner et al., 1996). The predictability and frequency effects suggest that a skipped word has already been processed to a certain degree while the eyes were fixating on the previous word. Considerable debate in the eye movement literature focuses precisely on the extent to which a word can be processed in parafoveal vision and what effects this has 
on eye movement control (e.g. Radach \& Kennedy, 2004). Whereas some models posit that the eye movement system can skip a word only when the word has been completely recognized on the prior fixation or when full recognition is imminent (e.g. the E-Z Reader model, Reichle, Rayner, \& Pollatsek, 2003), other models claim that word skipping is based on coarser information and that it entails an educated guess, taking into account factors such as word length and only very partial word identification. In the EOVP model (Brysbaert \& Vitu, 1998), for instance, the main determiners of the decision to skip a word are the length of the word and the experience the system has built up with how often a word of a certain length at a certain distance can be skipped without hindering overall text comprehension. This decision can be made with very limited information about the identity of the word.

Other models, such as the SWIFT model (Engbert, Longtin, \& Kliegl, 2002; Engbert, Nuthmann, Richter, \& Kliegl, 2005) or the Glenmore model (Reilly \& Radach, 2003, 2006) can be placed somewhere between the two views mentioned above in terms of how much parafoveal processing they assume to take place prior to skipping. In the SWIFT model, the lexical processing associated with a word is assumed to build up gradually until it has reached a maximum value, after which the lexical activity associated with that word declines. Saccades will be oriented towards words that have the highest level of excitation, which occurs at the peak of lexical processing of that word. As a consequence, the more processing of the parafoveal word (word $n+1$ ) has occurred, the higher the chances that the level of excitation for this word will already have passed its peak and be surpassed by the level of excitation of the subsequent word (word $n+2$ ). Word $n+2$ will then win the competition of becoming the target for the next saccade and word $n+1$ will be skipped. As a consequence, SWIFT allows a word to be skipped even when the level of lexical processing of word $n+1$ has not yet reached the amount assumed by the E-Z Reader model (e.g. a word $n+2$ with a very high level of excitation would increase the chances of the word $n+1$ being skipped, regardless of the level of excitation associated with word $n+1)$. On the other hand, SWIFT usually assumes much more processing of word $n+1$ before it can be skipped than is assumed in the EOVP model.

It is important to stress that most studies that find linguistic influences on word skipping did control for visual factors and vice versa (Brysbaert, Drieghe, \& Vitu, 2005). Hence, any comprehensive model on word skipping will have to take into account both visual and linguistic factors. For instance, in the E-Z Reader model the effect of word length on skipping is incorporated because the model assumes an inverse relation between the extraction of letter information and the distance of a letter from the centre of the visual field. Thus, the further away the eyes are from the target word, the more time will be needed to complete the first phase of word recognition. Since word skipping depends on word recognition, this slowdown in the word recognition process leads to a smaller chance that the word will be skipped. Basically the same principle features in the SWIFT model. Owing to reduced efficiency of information extraction of a word at a large eccentricity, the chances are lower that this word will have passed its peak in excitation. Likewise, the EOVP model incorporates (limited) linguistic influences on skipping behaviour. Whereas the EOVP model states that the initial decision whether or not to skip the next word will be based on word length and the distance of the word from the current fixation location, it does allow some limited saccade target adaptation by incoming linguistic information after this initial decision to skip or not has been made. 
A convincing illustration of the importance of incorporating both sources of influence on word skipping is provided by Drieghe, Rayner, and Pollatsek (2005). In their first experiment they showed that a high amount of lexical processing can influence the decision of the eye movement system to skip a word. In a sentence such as 'The doctor told Fred that his drinking would damage his liver very quickly', the word liver is highly predictable from the preceding context. The skipping rates of this predictable target word were compared with sentences in which the target word was replaced either by a neutral word (e.g. heart) or by an illegal non-word that was visually identical to the predictable word with the exception of a single letter (e.g. livor). Drieghe et al. showed that the predictable word was skipped more often than the neutral word, replicating the findings by Balota et al. (1985). However, no difference was reported between the skipping rates of the neutral word (heart) and those of the visually similar non-word (livor). In other words, only if the word completely matched the predictable word did an effect of predictability on skipping rate emerge. However, in a second experiment, evidence was found that orthographically illegal non-words (e.g. hxt) were skipped too often to be attributed to factors such as saccadic error (at a close launch site $31 \%$ of the orthographically illegal non-words were skipped). ${ }^{1}$ If the decision to skip was exclusively determined by the success of recognizing the word in the parafovea, non-words would not be skipped so often. In all likelihood, factors that are more low-level than the lexical processing of the parafoveal word (such as the short length of these orthographically illegal non-words) were playing an important role in causing the eyes to skip these non-words. Thus, from these two experiments, it seems that both visual and linguistic factors play an undeniable role in determining skipping behaviour. It becomes clear that the decision of which theoretical model gives the best account on how the system arrives at skipping words, will be answered on the basis of the relative importance of these factors instead of choosing one type of factor over the other.

In an attempt to gain a more accurate view of the relative importance of visual vs. linguistic influences on word skipping, Brysbaert et al. (2005; for an earlier version see Brysbaert \& Vitu, 1998) carried out a meta-analysis of all word-skipping studies that manipulated the processing ease of words and that reported word lengths. A distinction was made between a first group of studies that manipulated the processing ease of the target word in terms of word characteristics (e.g. the frequency of the word) and a second group of studies that manipulated processing ease in terms of contextual predictability. For the first group of studies Brysbaert et al. reported a consistent 5\% difference in skipping rate between the easy and the difficult words, whereas the wordlength effect ranged from a negligible $2 \%$ skipping rate for nine-letter words to over $50 \%$ skipping rates for two-letter target words. For the second group of studies the average effect of contextual predictability on word skipping amounted to an $8 \%$ difference between the predictable target words and the neutral words. The effects of word length were identical to those found for the first group of studies. From these findings the authors concluded that to predict the skipping rate of a word, it was more useful to know how long it is than how easy it is to process.

Given the theoretical importance of these data, it is interesting to look at the range of effects of linguistic factors on word skipping. Whereas the meta-analysis revealed very

\footnotetext{
'Targeting errors are far from rare. A recent analysis by Nuthmann, Engbert, and Kliegl (2005) suggests that more than 10\% of saccades miss their intended target.
} 
similar ranges of processing ease effects in the studies that manipulated word characteristics (effects of $0-13 \%$ ) and the studies that manipulated context predictability (effects of $0-12 \%$ ), there was one major exception in the latter group of studies. Vonk (1984) reported a difference of $23 \%$ in skipping rate between the predictable and neutral condition; almost twice the effect of the largest skipping rate of $12 \%$ and almost three times as much as the average skipping rate of $8 \%$. This is the only study that reports a linguistic effect that appears to be comparable in size with the visual word-length effects. Therefore, it is crucial for our understanding of the role of linguistic and visual factors in word skipping (and eye movement behaviour in general) to look more closely at this experiment.

Vonk (1984) compared the skipping rates of Dutch pronouns in sentences such as (1):

(1a) Alex beloog Andy omdat hij onraad rook [Alex lied to Andy because he smelled trouble.]

(1b) Alex beloog Anna omdat hij onraad rook.

[Alex lied to Anna because he smelled trouble.]

Vonk investigated whether readers are able to process a sentence in a selective way; i.e. whether they pay more attention to words if they contain new information than if their meaning is made redundant by the preceding clause. In sentence (1a), where two masculine names are used, the pronoun be does not provide disambiguation information concerning the intended antecedent of the pronoun. In sentence (1b), where a masculine and feminine name are used, the gender of the pronoun is informative as to which entity in the previous clause is the correct antecedent of the pronoun. Vonk reported a skipping rate of $40 \%$ of the pronoun in sentence (1a) and $17 \%$ in sentence $(1 \mathrm{~b})$. She interpreted these skipping rates as evidence for a rational selection of information in sentence reading. Given that the pronoun did not offer any extra information in terms of the correct antecedent of the pronoun in sentence (1a), it was skipped more often than in sentence (1b), where the pronoun was informative.

However, whereas most studies use a normal reading task to investigate skipping behaviour, Vonk (1984) used a referent naming task, in which participants had to vocalize the intended antecedent of the pronoun. Participants pressed a button in order for a sentence to appear on the screen. Their task was to name the correct referent for the pronoun as quickly as possible. This means that the participant could name the antecedent of the pronoun before they had read the entire sentence. After the participant named the referent of the pronoun, he/she had to press the button again to end the trial. It is not inconceivable that participants quite quickly grasped that a combination of two antecedents sharing the same gender made the identity of the pronoun referentially ambiguous and, therefore, useless for the task at hand (naming the intended antecedent). On the other hand, when one of the names is masculine and one is feminine, it is easy to see that simply looking at the pronoun would immediately provide you with the right response to the task (leading to almost no skipping in that case). Consequently, it is very likely that the task that Vonk used, exaggerated the difference between fixating ambiguous and unambiguous pronouns. Therefore, and especially given the rather special position this study holds in reported effects of linguistic factors on word skipping, it would be worthwhile to examine whether this effect would occur in the same size in an experiment using a task that more closely resembles normal reading. If the size of the pronoun ambiguity effect in normal reading 
is reduced to the $0-12 \%$ range - the range reported in all the other word-skipping studies looking at linguistic variables - this would be important for the discussion on the relative importance of visual vs. linguistic factors on word skipping. Finding out the actual size of the effect would also be important for people trying to model skipping data, as the size of the effect reported by Vonk clearly constitutes a statistical outlier. If, on the other hand, the size of the effect is upheld under normal reading conditions, this would shed new light on how pronoun resolution can effect word skipping. Similar to the original experiment, the current experiment was conducted in Dutch. This language has the extra advantage for word skipping research in that the male pronoun bij (he), has the same number of letters as the female pronoun $z i j$ (she), which permits perfect matching on word length.

\section{Method}

\section{Participants}

Thirty-six members of the Ghent University community participated in this experiment. All participants were native speakers of Dutch and had normal or corrected-to-normal vision. They were paid $10 €$ for their participation.

\section{Apparatus}

Eye movements were recorded by an Eyelink I (SR Research, Toronto) video-based pupiltracking system. Viewing was binocular but eye movements were recorded from the right eye only. A high-speed video camera was used for recording. It was positioned underneath the monitored eye and held in place by head-mounted gear. The system has a visual resolution of 20 seconds of arc. Fixation locations were sampled every 4 milliseconds and these raw data were used to determine the different measures of oculomotor activity during reading. The display was 69 centimetres from the subject's eye and three characters equalled $1^{\circ}$ of visual angle. A chin rest was used to reduce head movements during the experiment.

\section{Materials}

Thirty-two target sentences were created. ${ }^{2}$ Each target sentence had two versions based on the disambiguating value of the pronoun: the gender of the pronoun was either ambiguous (both antecedents had the same gender as the pronoun) or unambiguous (the two antecedents had a different gender and thus the gender of only one antecedent corresponded to the gender of the pronoun). Half of the items used the masculine pronoun ( $h i j$ [he]), whereas the other half of the items used the feminine pronoun (zij [she]). We included both the masculine pronoun $b i j$ and the feminine pronoun $z i j$, because using only one of them could lead to participants developing a strategy in the unambiguous cases, in which, as soon as the two names are seen the correct antecedent is known (independent of the information carried by the pronoun). An example of the two conditions, both with a male and a female pronoun, is given in Table 1 together with their translations in English.

\footnotetext{
${ }^{2}$ All materials are available from the first author upon request, denis.drieghe@ugent.be.
} 
Table I. Examples of the sentences used in the experiment as a function of pronoun gender (masculine vs. feminine) and gender cue (ambiguous vs. unambiguous)

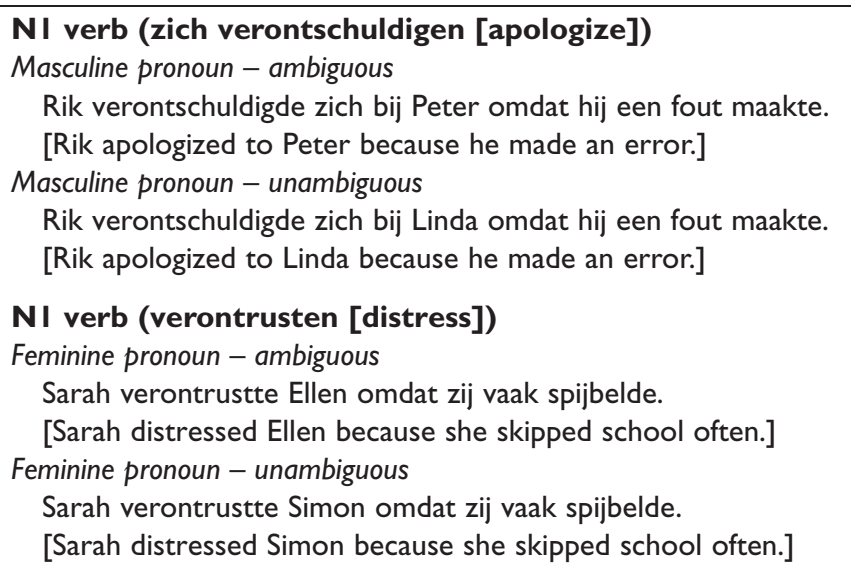

It is important to note that the type of material used by Vonk (1984) has subsequently mainly been used for the study of the role of implicit causality on pronoun resolution. The implicit causality bias is a semantic characteristic of verbs such that event descriptions (based on these verbs) sometimes implicitly indicate the cause of the event (e.g. Garnham, Oakhill, \& Cruttenden, 1992; Garnham, Traxler, Oakhill, \& Gernsbacher, 1996; Greene \& McKoon, 1995; Long \& De Ley, 2000; McDonald \& MacWhinney, 1995; McKoon, Greene, \& Ratcliff, 1993). For instance, when participants are presented with the sentence 'John amazed Mary' and are asked to indicate the cause of this event, they will predominantly attribute the cause to the agent of the event (i.e. the first-mentioned name in active sentences, such as John). However, when participants are presented with the sentence 'John admired Mary', they will predominantly attribute the cause to the patient of the event (i.e. the secondmentioned name in active sentences, such as Mary). For this reason, verbs such as amaze are called N1 verbs and verbs such as admire are called N2 verbs. The implicit causality bias also has an online effect in that sentences which contain an explicit cause that is congruent with the implicit bias (e.g. 'John admired Mary because she was a very smart woman') are read faster than sentences which contain an explicit cause that is incongruent with the implicit bias (e.g. 'John admired Mary because he liked very smart women') (Stewart, Pickering, \& Sanford, 2000). In order to avoid any confounding effects of this much-studied implicit causality bias in our study, we made sure to have as many N1-biased verbs as N2-biased verbs in our stimuli, and that the explicit cause in our items was always congruent with the implicit causality bias.

\section{Procedure}

Before the experiment started, participants were informed that the study was about the comprehension of sentences which were displayed on a computer screen. Sentence administration was self-paced. Participants started and stopped sentence presentation by pressing on a button. Each sentence was presented as a whole. Participants were asked to read at their normal speed, and to answer any comprehension question that followed the sentence. Questions, which were simple true/false statements, followed 
on a quarter of the trials. The participants had no difficulty answering these questions (the overall question answering accuracy rate was 96\%). The initial calibration of the eye-tracking system generally took approximately 10 minutes and consisted of a standard nine-point grid. Following the initial calibration the participant was given 10 practice trials to become familiar with the procedure before reading the experimental sentences. The 32 experimental sentences were embedded in a pseudo-random order in 110 filler texts. These filler texts were part of other reading experiments conducted at our laboratory and could consist of either a single sentence or a fragment of text. The fragments of text were always presented as a whole. Each participant was presented with one of the two possible variants of the critical text fragments according to a Latin square design. Participants completed a single session containing 142 text fragments to read in approximately 50 minutes.

\section{Results}

Our primary interest in this experiment was the probability of skipping the pronoun during the first pass through the text. In addition to the skipping probability, we also examined the fixation times on the pronoun. Owing to the fact that the pronoun was a three-letter word, it is not surprising that when the pronoun was fixated it received only a single fixation in $98 \%$ of the cases. Therefore, we restricted the fixation duration analyses on the pronoun to those occasions when there was a single fixation. Besides fixation times on the pronoun we also examined the first-pass time of the region after the pronoun, which was defined as the two words following the pronoun, to see whether the effect of gender cue resulted in any differences in eye movements after the pronoun was or was not skipped. The first-pass time is the sum of all the fixations on a region prior to an eye movement to another region in the text. The overall reading times were also computed in order to establish whether one type of sentence was more difficult to read than the other. Only $1.8 \%$ of the data were removed from the analyses because of track loss or because the fixation was shorter than 100 milliseconds (see Morrison, 1984; Rayner, Sereno, Morris, Schmauder, \& Clifton, 1989, for justification). All analyses were run over participants (F1-analyses) and items (F2-analyses).

\section{Skipping the pronoun}

The skipping probabilities of the pronoun are shown in Table 2.

Table 2. Skipping probability of the pronoun and fixation times on the pronoun, the first-pass time of the region after the pronoun and the total reading times for the entire sentences as a function of gender cue and the gender of the pronoun

\begin{tabular}{lccrrrr}
\hline & \multicolumn{2}{c}{ Gender cue } & & \multicolumn{2}{c}{ No gender cue } \\
\cline { 2 - 3 } & hij (he) & zij (she) & & hij (he) & zij (she) \\
\hline Skipping probability of the pronoun & $51 \%$ & $46 \%$ & & $60 \%$ & $45 \%$ \\
Single fixation time on the pronoun (ms) & 210 & 212 & & 208 & 214 \\
First pass time of the region after the pronoun (ms) & 352 & 410 & & 340 & 394 \\
Total reading time of the sentences (ms) & 2396 & 2489 & & 2382 & 2497 \\
\hline
\end{tabular}


We ran a repeated-measures ANOVA on the skipping probabilities of the pronoun with gender cue (presence vs. absence of a gender cue) and the gender of the pronoun (he or she) as independent variables. ${ }^{3}$ The $5 \%$ effect of gender cue was not significant $[F 1(1,35)=2.14, p>.10 ; F 2(1,15)<1, n s]$ but the effect of pronoun gender was significant $[F 1(1,35)=7.90, p<.01 ; F 2(1,15)=9.68, p<.01]$. On average the male pronoun ( $b i j$, he) was skipped $10 \%$ more often than the female pronoun (zij, she). There was no significant interaction between these two factors $[F 1(1)=$,2.10 , $p>.10 ; F 2(1,15)=2.77, p>.10]$. However, to make sure that we did not miss any effect of the gender cue we analyzed the skipping probabilities of the 'he' and 'she' conditions separately. These planned comparisons showed that the pronoun 'he' was skipped 9\% more often when there was no gender cue than when there was one $[t 1(35)=2.07, p<.05 ; t 2(35)=2.77, p=.052]$. The same effect was not present in the 'she' conditions (all $t<1$ ).

\section{Single fixation times on the pronoun}

As can be seen from the fixation times shown in Table 2 there was no effect of gender cue (all $F<1)$ and no effect of the pronoun gender on the single fixation times on the pronoun $($ all $F<1$ ). The interaction between these variables was not significant either (all $F<1$ ).

\section{First-pass time on the region after the target}

The effect of gender cue was not significant on the first-pass time of the region after the target $[F 1(1,35)=2.58, p>.10 ; F 2(1,15)=1.63, p>.20]$. On the other hand, the effect of pronoun gender was significant in the participants' analysis $[F 1(1,35)=32.08, p<.001]$ but not in the item analysis $[F 2(1,15)=2.79$, $p>$.10]. Thus, there was some indication that the region following the target word was read faster in the 'he' conditions than in the 'she' conditions. The interaction between these two factors was not significant (all $F<1$ ).

\section{Total reading times of the sentences}

The effect of gender cue was not significant in the analyses of the total reading times (all $F<1$ ) but there was a significant effect of pronoun gender, although again restricted to the participants' analysis $[F 1(1,35)=7.47, p<.01 ; F 2(1,15)=1.44, p>.20]$. The sentences containing a 'he' pronoun appear to be read on average 104 milliseconds faster than the sentences containing a 'she' pronoun. The interaction between these two factors was not significant (all $F<1$ ).

\section{Fixation prior to the target}

Given the rather surprising finding that our results were different when the pronoun was masculine compared with when it was feminine, we conducted an analysis on the fixation duration prior to either the landing on or the skipping of the pronoun. In other words, we analysed the fixation times on the last fixation left

\footnotetext{
${ }^{3}$ Since we made sure that the antecedent of the pronoun was always congruent with the NI or N2 implicit causality bias of the verb, this factor did not cause any significant effects throughout all the analyses reported here (all $F<I)$. Therefore, this factor was not taken into account while reporting the other analyses.
} 
to the pronoun. Although unlikely, should any differences between the stimulus sets containing the masculine or the feminine pronoun contribute to our skipping data, these differences would most certainly be apparent in these analyses. These analyses could also be informative whether any of our manipulations had an effect prior to our region of interest, the pronoun. Table 3 reports both the mean fixation duration and the launch site as a function of whether the next saccade either lands on the pronoun or skips it.

Table 3. Mean fixation duration and launch site (distance from the pronoun in character positions) prior to the skipping of or the landing on the pronoun as a function of gender cue and the gender of the pronoun

\begin{tabular}{lccccr}
\hline & \multicolumn{2}{c}{ Gender cue } & & \multicolumn{2}{c}{ No gender cue } \\
\cline { 2 - 3 } & hij (he) & zij (she) & & hij (he) & zij (she) \\
\hline Fixation duration prior to skipping (ms) & 223 & 225 & & 220 & 226 \\
Fixation duration prior to landing (ms) & 234 & 240 & & 229 & 239 \\
Launch site prior to skipping & 3.7 & 3.2 & & 3.9 & 4.0 \\
Launch site prior to landing & 8.2 & 7.4 & & 7.9 & 7.5 \\
\hline
\end{tabular}

As is apparent from the means, the analysis of the fixation duration prior to skipping showed no effects of gender cue, pronoun gender or an interaction between these two variables (all $F<1$ ). The same pattern was observed for the fixation duration prior to landing (all $F<1$ ). For the analysis of the launch site prior to the skipping of the pronoun there was no effect of gender cue $(F 1(1,28)=1.67, p>.20 ; F 2(1,15)=1.93, p>.10)$ and no effect of pronoun gender (all $F<1$ ). The interaction between these two factors was also not significant (all $F<1$ ). The analysis of the launch site prior to landing also did not result in any significant differences: there was no effect of gender cue (all $F<1$ ), no effect of pronoun gender $(F 1(1,31)=2.48, p>.10 ; F 2<1, n s)$ and the interaction was also not significant $(F 1(1,31)<1, n s ; F 2(1,15)=2.40, p>.10)$. Collapsing over all the data, we did replicate the well-documented effect of launchsite: the eyes were four character positions closer to the pronoun when they skipped the pronoun than when they did not (respectively 3.7 character positions vs. 7.7 character positions, $t 1(35)=-14.74, p<.001 ; t 2(31)=-11.86, p<.001)$. The fixation duration prior to skipping was also 15 milliseconds less than prior to landing on the pronoun, although this effect was only marginally significant in the item analysis (respectively 223 vs. 238 milliseconds, $t 1(34)=3.44, p<.01$; $t 2(31)=1.92, p=.06){ }^{4}$

\footnotetext{
${ }^{4}$ Looking at the launch-site analyses, it is apparent that the saccades resulting in the skipping of the pronoun more often came from the word prior to the pronoun (i.e. omdat [because]) than was the case when the saccade resulted in landing on the pronoun. In all likelihood the reduced fixation duration prior to skipping reflects the high frequency of the word omdat [because]. Recently, there has been much discussion in the literature on whether or not the fixation duration prior to skipping is inflated (e.g. Drieghe et al., 2005). This observation is important because it is quite often seen as one of the ways to distinguish between the different models of eye movement control in reading (for a discussion see Kliegl \& Engbert, 2005). However, for the current study we do not have sufficient data, hence statistical power, to conduct an analysis of the fixation duration prior to skipping/landing that is sufficiently controlled for the launch site. Therefore we will look no further into this issue.
} 


\section{Discussion}

The main goal of the current study was to test whether the effect size of a linguistic factor (i.e. a gender cue) on the skipping rate of pronouns, as reported by Vonk (1984), could be replicated in a task that more closely resembles normal reading. Vonk reported that a gender-marked pronoun was skipped $23 \%$ more often when the two potential antecedents had the same gender than when the two antecedents were of a different gender. The size of this effect is quite unusual, given that a recent meta-analysis (Brysbaert et al., 2005) showed that all other reported effects of a linguistic origin on word skipping were in the range of $0-13 \%$. Therefore, it was important to re-examine this study as it clearly constitutes an outlier in terms of linguistic influences on word skipping. If the size of the effects in normal reading are of a comparable magnitude to those observed in the original study, this would show a stronger influence of linguistic factors on the decision to skip a word than previously observed. It would mean that at least some linguistic factors (pronoun ambiguity) can strongly influence skipping behaviour. It would also mean that word skipping is very instrumental in examining early effects of pronoun resolution, because one of the major debates in the psycholinguistic literature on implicit causality bias is exactly about how early this bias can influence pronoun resolution (e.g. Stewart et al., 2000). However, there were $a$ priori reasons to doubt whether we would replicate such a large effect in normal reading. The task in the Vonk experiment was to vocalize as quickly as possible the name of the antecedent of the pronoun. It is not inconceivable that this task elicited different processing strategies (focusing more on the gender information of the pronoun) than those used in other reading experiments investigating the effect of linguistic variables on word skipping. In the current study the task was similar to other word-skipping studies, namely reading for comprehension.

Our results clearly show that the large effects of gender cue on the skipping probability of the pronoun reported by Vonk (1984) were a consequence of the task used. Using the same type of materials, we found a non-significant effect of $5 \%$ more skipping of the pronoun when it was uninformative than when it was informative. At the same time, there seemed to be an unexpected difference between the masculine pronoun $b i j$ and the feminine pronoun $z i j$. The pronoun $b i j$ was skipped $10 \%$ more often than the pronoun $z i j$ and showed the clearest evidence for an effect of gender cue ( 9 vs. $1 \%$ for $z i j)$. Moreover, the latter effect was statistically significant, replicating the findings reported by Vonk for the male pronouns, but bringing them within the range of effect sizes found with other linguistic variables. Also, from the reading times, it was clear that sentences with the pronoun $b i j$ were easier to understand than sentences with the pronoun $z i j$.

The fact that we replicated the effect due to the pronoun informativeness, even though in a much less dramatic way than in the original Vonk (1984) study, indicates that readers sometimes look at parts of a sentence in a selective way. In this experiment they preferred to move the eyes further into the text if the gender of the antecedents made the pronoun redundant. Note that this is very likely an effect of strategically positioning the eyes and is not solely due to the processing ease of the pronoun itself. The fact that no effects whatsoever were observed in the fixation times of the pronoun as a function of our manipulations shows that the skipping rates in this experiment do not merely reflect the processing ease of the pronoun (although this lack of effects could also originate from a floor effect given the very short fixation times). When a word is redundant, readers skip the word more often so that they can position the eyes further in the text. Before dealing with the difference we observed as a function of the gender of 
the pronoun, we first discuss the implications of the removal of the Vonk data from the charts of effects on word skipping in normal reading.

Theoretical accounts of the word-skipping phenomenon differ greatly in the extent to which they allow parafoveal preprocessing to determine the target of a between-word saccade. Models such as the E-Z Reader model (Reichle et al., 2003) state that in order for a word to be skipped the system either must have recognized the word on the prior fixation or that full recognition must be imminent. Other models, such as the EOVP model (Brysbaert \& Vitu, 1998), claim that word skipping is primarily based on coarse information concerning the parafoveal word, such as its length and its distance from the current fixation position. Only in a second stage can parafoveal word processing affect the initial decision, either by cancelling an intended saccade to the next word or by cancelling the initial decision to skip the next word. Without going into the details of these models, it is important to note that all current models that make explicit claims on word skipping in reading incorporate influences of both a linguistic and a visual nature. Thus, the main argument is about which of the influences on word skipping is the main player in determining the decision whether or not to skip the following word. If low-level visual factors (e.g. word length) are the main players, this would be a strong indication that the (initial) decision to skip a word is primarily based on relatively coarse information concerning that word. If, on the other hand, linguistic factors are the most important factor in determining skipping behaviour, this would clearly indicate that it is the advanced state of recognizing the parafoveal word that triggers the word-skipping saccade.

Our present findings constitute an important addition to the meta-analysis reported by Brysbaert et al. (2005), which looked at the relative importance of visual vs. linguistic factors in determining word-skipping behaviour. This meta-analysis showed that in order to predict the skipping rate of a word it was more informative to know the word length than to know how easy the word was to process. One study did not fit that picture: Vonk (1984) reported an effect of a linguistic origin that was comparable in size to the reported word-length effects. By removing this study from the charts we add further evidence to the claim that linguistic influences are not the main determinant of the decision to skip the upcoming word. Although word skipping is affected by the informativeness and the difficulty of the word, it is more informative to know the length of the parafoveal word in order to predict whether it will be skipped or not.

Our results also suggested, although this interaction was not significant, that the effect of gender cue was restricted to the skipping of the male pronoun $b i j$ (he). This finding was quite surprising since no such effects were observed in the Vonk (1984) study or anywhere else in the literature. However, the presence of another unexpected finding, namely that the male pronoun $b i j$ (he) was, overall, skipped more frequently than the female pronoun $z i j$ (she) can point us in the direction of a plausible explanation. Note that we had included both pronouns simply to exclude the possibility that always the female (if we would have used only $z i j$ ) or male name (if we would have used only $\mathbf{h i j}$ ) would be the correct antecedent. Within a trial we always compared skipping of the same pronoun in the condition where both names had a different gender with the condition where both names had the same gender. We did not expect that this factor would have an influence on skipping, because both pronouns are the same length and are used extremely frequently (the pronoun $b i j$ [he] has a a log frequency per million words of 4.05 , the pronoun $z i j$ [she] has a log frequency per million words of 3.42, see Baayen, Piepenbrock, \& Van Rijn, 1993). However, a peculiarity in Dutch is that the female singular pronoun $z i j$ (she) is identical to the plural pronoun $z i j$ (they). In other words when readers encounter a sentence such as 'Laura verontschuldigde zich 
bij Simon omdat zij . . . [Laura apologized to Simon because she . . ]' it is perfectly legal to continue the sentence in such a manner that $z i j$ refers to both Laura and Simon (e.g. Laura verontschuldigde zich bij Simon omdat zij vroeger afgesproken hadden [Laura apologized to Simon because they arranged to meet earlier]). Owing to this peculiarity in Dutch it is not inconceivable that readers, upon encountering $z i j$, process both the singular female and the plural possibility. If this were the case, we would expect to observe in the current experiment both lower skipping rates for the pronoun $z i j$ (she) and somewhat longer total reading times in the $z i j$ (she) sentences, indicating the higher processing costs associated with processing both the female singular and the plural option. We observed indications of both these predictions in the current experiment (although one has to be very careful with interpreting the total reading times, given the fact that pronoun gender was manipulated across two halves of the stimulus set). This phenomenon is clearly beyond the scope of the present study and we can only offer a post boc interpretation that should be properly tested by matching the sentences used for the pronouns. However, we do see this explanation as the most plausible cause both for the lower skipping rates we observed in the female pronoun conditions and for the absence of the gender cue effect in those conditions: the fact that $z i j$ could refer to both a female singular antecedent and plural antecedents would in all likelihood destroy any effect of redundancy we could observe in these conditions.

To summarize, in this study we re-examined a prior experiment (Vonk, 1984) that was the only one to report an effect of a linguistic nature on word-skipping rates, which was in size comparable to the effects reported for word length. Using a task more closely resembling normal reading, we were able to show that the size of this effect was well within the range of previously reported effect sizes of linguistic factors in word skipping. The predictability effect was 5\% if we looked at all the sentences of the type used by Vonk, and $9 \%$ if we only looked at the sentences for which there was no discussion about the distinction between informative and uninformative pronouns. Whereas these findings in themselves clearly constitute an example of a linguistic influence on word skipping during reading, the effect sizes observed in the current experiment are considerably smaller than those commonly reported in studies as a consequence of manipulating the word length of the word that is being skipped (Brysbaert et al., 2005). From this perspective, these findings also corroborate previously made statements, that in order to predict whether a word will be skipped or not, it is more instrumental to know the length of the word than its processing ease.

\section{Acknowledgements}

Denis Drieghe and Timothy Desmet are postdoctoral fellows of the Fund for Scientific Research (Flanders, Belgium). We thank Barbara Juhasz, Simon Liversedge and Françoise Vitu for many helpful comments on previous drafts of this article.

\section{References}

Baayen, R. H., Piepenbrock, R., \& Van Rijn, H. (1993). The Celex Lexical Database (CD-ROM). Philadelphia, PA: Linguistic Data Consortium, University of Pennsylvania.

Balota, D. A., Pollatsek, A., \& Rayner, K. (1985). The interaction of contextual constraints and parafoveal visual information in reading. Cognitive Psychology, 17, 364-390.

Binder, K. S., Pollatsek, A., \& Rayner, K. (1999). Extraction of information to the left of the fixated word in reading. Journal of Experimental Psychology: Human Perception and Performance, 25, 1162-1172. 
Brysbaert, M., Drieghe, D., \& Vitu, F. (2005). Word skipping: Implications for theories of eye movement control in reading. In G. Underwood (Ed.), Cognitive processes in eye guidance (pp. 53-77). Oxford: Oxford University Press.

Brysbaert, M., \& Vitu, F. (1998). Word skipping: Implications for theories of eye movement control in reading. In G. Underwood (Ed.), Eye guidance in reading and scene perception (pp. 125-147). Oxford: Elsevier.

Drieghe, D., Brysbaert, M., \& Desmet, T. (2005). Parafoveal-on-foveal effects on eye movements in text reading: Does an extra space make a difference? Vision Research, 45, 1693-1706.

Drieghe, D., Brysbaert, M., Desmet, T., \& Debaecke, C. (2004). Word skipping in reading: On the interplay of linguistic and visual factors. European Journal of Cognitive Psychology, 16, 79-103.

Drieghe, D., Rayner, K., \& Pollatsek, A. (2005). Word skipping during reading revisited. Journal of Experimental Psychology: Human Perception and Performance, 31, 954-969.

Ehrlich, S. F., \& Rayner, K. (1981). Contextual effects on word perception and eye movements during reading. Journal of Verbal Learning and Verbal Behaviour, 20, 641-655.

Engbert, R., Longtin, A., \& Kliegl, R. (2002). A dynamical model of saccade generation in reading based on spatially distributed lexical processing. Vision Research, 42, 621-636.

Engbert, R., Nuthmann, A., Richter, E. M., \& Kliegl, R. (2005). SWIFT: A dynamical model of saccade generation during reading. Psychological Review, 112, 777-813.

Garnham, A., Oakhill, J., \& Cruttenden, H. (1992). The role of implicit causality and gender cue in the interpretation of pronouns. Language and Cognitive Processes, 7, 231-255.

Garnham, A., Traxler, M., Oakhill, J., \& Gernsbacher, M. A. (1996). The locus of implicit causality effects in comprehension. Journal of Memory and Language, 35, 517-543.

Greene, S. B., \& McKoon, G. (1995). Telling something we can't know: Experimental approaches to verbs exhibiting implicit causality. Psychological Science, 6, 262-270.

Henderson, J. M., \& Ferreira, F. (1993). Eye movement control during reading: Fixation measures reflect foveal but not parafoveal processing difficulty. Canadian Journal of Experimental Psychology, 47, 201-221.

Inhoff, A. W., \& Rayner, K. (1986). Parafoveal word processing during eye fixations in reading: Effects of word frequency. Perception and Psychophysics, 40, 431-439.

Kerr, P. W. (1992). Eye movement control during reading: The selection of where to send the eyes. Doctoral dissertation, University of Illinois at Urbana-Champaign, Urbana, IL.

Kliegl, R., \& Engbert, R. (2005). Fixation durations before word skipping in reading. Psychonomic Bulletin and Review, 12, 132-138.

Liversedge, S. P., \& Findlay, J. M. (2000). Saccadic eye movements and cognition. Trends in Cognitive Sciences, 4, 6-14.

Long, D. L., \& De Ley, L. (2000). Implicit causality and discourse focus: The interaction of text and reader characteristics in pronoun resolution. Journal of Memory and Language, 42, 545-570.

McDonald, J. L., \& MacWhinney, B. (1995). The time course of anaphor resolution: Effects of implicit verb causality and gender. Journal of Memory and Language, 34, 543-566.

McKoon, G., Greene, S. B., \& Ratcliff, R. (1993). Discourse models, pronoun resolution, and the implicit causality of verbs. Journal of Experimental Psychology: Learning, Memory, and Cognition, 19, 1040-1052.

Morrison, R. E. (1984). Manipulation of stimulus onset delay in reading: Evidence for parallel programming of saccades. Journal of Experimental Psychology: Human Perception and Performance, 10, 667-682.

Nuthmann, A., Engbert, R., \& Kliegl, R. (2005). Mislocated fixations during reading and the inverted optimal viewing position. Vision Research, 45, 2207-2217.

Radach, R., \& Kempe, V. (1993). An individual analysis of initial fixation positions in reading. In G. d'Ydewalle \& J. Van Rensbergen (Eds.), Perception and cognition: Advances in eye movement research (pp. 213-226). Amsterdam: North Holland. 
Radach, R., \& Kennedy, A. (2004). Theoretical perspectives on eye movements in reading: Past controversies, current issues, and an agenda for future research. European Journal of Cognitive Psychology, 16, 3-26.

Rayner, K. (1978). Eye movements in reading and information processing. Psychological Bulletin, $85,618-660$.

Rayner, K. (1979). Eye guidance in reading: Fixation locations within words. Perception, 8, 21-30.

Rayner, K. (1998). Eye movements in reading and information processing: 20 years of research. Psychological Bulletin, 124, 372-422.

Rayner, K., Binder, K. S., Ashby, J., \& Pollatsek, A. (2001). Eye movement control in reading: Word predictability has little influence on initial landing positions in words. Vision Research, 41, 943-954.

Rayner, K., \& Duffy, S. A. (1986). Lexical complexity and fixation times in reading: Effects of word frequency, verb complexity, and lexical ambiguity. Memory and Cognition, 14, 191-201.

Rayner, K., \& Fischer, M. H. (1996). Mindless reading revisited: Eye movements during reading and scanning are different. Perception and Psychophysics, 58, 734-747.

Rayner, K., \& McConkie, G. W. (1976). What guides a reader's eye movements? Vision Research, $16,829-837$

Rayner, K., Sereno, S. C., Morris, R. K., Schmauder, A. R., \& Clifton, C. (1989). Eye movements and on-line language comprehension processes. Language and Cognitive Processes, 4, 1-50.

Rayner, K., Sereno, S. C., \& Raney, G. E. (1996). Eye movement control in reading: A comparison of two types of models. Journal of Experimental Psychology: Human Perception and Performance, 22, 1188-1200.

Rayner, K., \& Well, A. D. (1996). Effects of contextual constraint on eye movements during reading: A further examination. Psychonomic Bulletin and Review, 3, 504-509.

Reichle, E. D., Rayner, K., \& Pollatsek, A. (2003). The E-Z Reader model of eye movement control in reading: Comparisons to other models. Behavioral and Brain Sciences, 26, 445-526.

Reilly, R. G., \& Radach, R. (2003). Foundations of an interactive model of eye movement control in reading. In J. Hyönä, R. Radach, \& H. Deubel (Eds.), Cognitive and applied aspects of eye movement research (pp. 429-455). Amsterdam: Elsevier.

Reilly, R. G., \& Radach, R. (2006). Some empirical tests of an interactive activation model of eye movement control in reading. Cognitive Systems Research, 7, 34-55.

Schilling, H. E. H., Rayner, K., \& Chumbley, J. I. (1998). Comparing naming, lexical decision, and eye fixation times: Word frequency effects and individual differences. Memory and Cognition, 26, 1270-1281.

Starr, M. S., \& Rayner, K. (2001). Eye movements during reading: Some current controversies. Trends in Cognitive Sciences, 5, 156-163.

Stewart, A. J., Pickering, M. J., \& Sanford, A. J. (2000). The time course of the influence of implicit causality information: Focusing versus integration accounts. Journal of Memory and Language, 42, 423-443.

Vitu, F. (1991). The influence of parafoveal processing and linguistic context on the optimal landing position effect. Perception and Psychophysics, 50, 58-75.

Vitu, F., O'Regan, J. K., Inhoff, A. W., \& Topolski, R. (1995). Mindless reading: Eye-movement characteristics are similar in scanning letter strings and reading texts. Perception and Psychophysics, 57, 352-364.

Vonk, W. (1984). Eye movements during comprehension of pronouns. In A. G. Gale \& F. Johnson (Eds.), Theoretical and applied aspects of eye movement research (pp.203-211). Amsterdam: North-Holland.

White, S. J., \& Liversedge, S. (2004). Orthographic familiarity influences initial eye fixation positions in reading. European Journal of Cognitive Psychology, 16, 52-78.

Received 8 November 2005; revised version received 21 March 2006 